TRANSACTIONS OF THE

AMERICAN MATHEMATICAL SOCIETY

Volume 360, Number 7, July 2008, Pages 3613-3630

S 0002-9947(08)04450-4

Article electronically published on February 27, 2008

\title{
GENERICALLY THERE IS BUT ONE SELF HOMEOMORPHISM OF THE CANTOR SET
}

\author{
ETHAN AKIN, ELI GLASNER, AND BENJAMIN WEISS
}

\begin{abstract}
We describe a self homeomorphism $R$ of the Cantor set $X$ and then show that its conjugacy class in the Polish group $H(X)$ of all homeomorphisms of $X$ forms a dense $G_{\delta}$ subset of $H(X)$. We also provide an example of a locally compact, second countable topological group which has a dense conjugacy class.
\end{abstract}

\section{INTRODUCTION}

A topological group $G$ is called Rohlin if there is an element $g \in G$ whose conjugacy class is dense in $G$. In [GW] it was shown that the Polish group $H(X)$ of homeomorphisms of the Cantor set $X$ is Rohlin. The same result was independently obtained in $\mathrm{AHK}$ and the authors there posed the question whether a much stronger property holds for $H(X)$, namely that there exists a conjugacy class which is a dense $G_{\delta}$ subset of $G$.

In A it was shown that the subgroup $G_{\mu}$ of the Polish group $G=H(X)$ of all homeomorphisms of the Cantor set $X$ which preserve a special kind of a probability measure $\mu$ on $X$ has the property that its action on itself by conjugation admits a dense $G_{\delta}$ conjugacy class. Recently this was shown by Kechris and Rosendal in KR to be the case for many other closed subgroups of $G=H(X)$, including $G$ itself. However the authors of $[\mathrm{KR}]$ use rather abstract model theoretical arguments in their proof and they present it as an open problem to give an explicit description of the generic homeomorphism.

In the present work we provide a new and more constructive proof of the Kechris and Rosendal result. We further supply a detailed description of the generic conjugacy class in $H(X)$. At the end of the paper we also provide an example of a locally compact, second countable Rohlin group $G$, i.e. $G$ has a dense conjugacy class. This answers a question of Kechris and Rosendal.

\section{Algebraic constructions}

Our spaces $X$ will be nonempty, compact and metrizable, e.g. compact subsets of $\mathbb{R}$, Cantor spaces and finite discrete spaces. For a homeomorphism $T: X \rightarrow X$ we will say that $T$ is a homeomorphism on $X$. A dynamical system is a pair $(X, T)$ with $T$ a homeomorphism on $X$.

Received by the editors April 26, 2006.

2000 Mathematics Subject Classification. Primary 22A05, 22D05; Secondary 54C40, 37E15.

Key words and phrases. Rohlin property, group of homeomorphisms of the Cantor set, conjugacy class.

This research was supported by ISF grant \# 1333/04.

(C) 2008 American Mathematical Society Reverts to public domain 28 years from publication 
Given dynamical systems $(X, T)$ and $\left(X_{1}, T_{1}\right)$ we say that a continuous function $F: X_{1} \rightarrow X$ is an action map from $\left(X_{1}, T_{1}\right)$ to $(X, T)$, or just $F$ maps $T_{1}$ to $T$, when $F$ is surjective and $F \circ T_{1}=T \circ F$. When such an $F$ exists we will say that $(X, T)$ is a factor of $\left(X_{1}, T_{1}\right)$ or just $T$ is a factor of $T_{1}$. A homeomorphism $F$ which maps $T_{1}$ to $T$ is called an isomorphism from $\left(X_{1}, T_{1}\right)$ to $(X, T)$ or from $T_{1}$ to $T$. In that case, $F^{-1}$ is an isomorphism from $T$ to $T_{1}$. Two systems are called isomorphic when an isomorphism between them exists.

Let $\mathbb{Z}$ denote the ring of integers and $\Theta_{m}$ denote the quotient ring $\mathbb{Z} / m \mathbb{Z}$ of integers modulo $m$ for $m=1,2, \ldots$. Let $\Pi: \mathbb{Z} \rightarrow \Theta_{m}$ denote the canonical projection. If $m$ divides $n$, then this factors to define the projection $\pi: \Theta_{n} \rightarrow \Theta_{m}$. The positive integers are directed with respect to the divisibility relation. We denote the inverse limit of the associated inverse system of finite rings by $\Theta$. This is a topological ring with a monothetic additive group on a Cantor space having projections $\pi: \Theta \rightarrow \Theta_{m}$ for positive integers $m$. We denote by $\Pi$ the induced map from $\mathbb{Z}$ to $\Theta$ which is an injective ring homomorphism. We also use $\Pi$ for the maps $\pi \circ \Pi: \mathbb{Z} \rightarrow \Theta_{m}$. Notice that we use $\pi$ for the maps with compact domain and $\Pi$ for the maps with domain $\mathbb{Z}$.

We can obtain $\Theta$ by using any cofinal sequence in the directed set of positive integers. We will usually use the sequence $\{k !\}$.

On each of these topological rings we denote by $\tau$ the homeomorphism which is translation by the identity element, i.e. $\tau(t)=t+1$. The dynamical system $(\Theta, \tau)$ is called the universal adding machine. The adjective "universal" is used because it has as factors periodic orbits of every period.

Let $\mathbb{Z}_{*}$ denote the two point compactification with limit points $\pm \infty$. Let $\tau$ be the homeomorphism of $\mathbb{Z}_{*}$ which extends the translation map by fixing the points at infinity. The points of $\mathbb{Z}$ form a single orbit of $\tau$ which tends to the fixed points in the positive and negative directions. We construct an alternative compactification $\Sigma$ of $\mathbb{Z}$ with copies of $\Theta$ at each end. $\Sigma$ is the closed subset of $\mathbb{Z}_{*} \times \Theta$ given by

$$
\Sigma={ }_{\text {def }} \quad\{(x, t): x= \pm \infty \quad \text { or } \quad x \in \mathbb{Z} \text { and } t=\Pi(x)\} .
$$

$\Sigma$ is invariant with respect to $\tau \times \tau$ and we denote its restriction by $\tau: \Sigma \rightarrow \Sigma$. A spiral is any dynamical system isomorphic to $(\Sigma, \tau)$. We will also refer to the underlying space as a spiral.

The points of $\{ \pm \infty\} \times \Theta$ are the recurrent points of the spiral. The remaining points, i.e. $\{(x, \Pi(x)): x \in \mathbb{Z}\}$ are the wandering points of the spiral.

We define the map $\zeta$ which collapses the spiral and identifies the ends:

$$
\zeta: \Sigma \rightarrow \Theta \quad \text { by } \quad \zeta(x, t)=t .
$$

That is, $\zeta$ is just the projection onto the second, $\Theta$, coordinate. Clearly, $\zeta:(\Sigma, \tau) \rightarrow$ $(\Theta, \tau)$ is an action map.

We will need finite approximations of the spiral.

If $W$ is a set, then a surjective relation $R$ on $W$ is a subset of $W \times W$ which maps onto $W$ via each projection. For example, a surjective map of $W$ is a surjective relation. The nonmap relations which we will need will be surjective relations on finite sets.

If $\phi: W_{1} \rightarrow W$ is a surjective set map and $R_{1} \subset W_{1} \times W_{1}$ is a surjective relation on $W_{1}$, then the image $R=\phi \times \phi\left(R_{1}\right) \subset W \times W$ is a surjective relation on $W$ and we say that $\phi$ maps $R_{1}$ to $R$. If $R_{1}$ and $R$ are surjective maps, then $\phi$ maps $R_{1}$ to $R$ iff $\phi \circ R_{1}=R \circ \phi$. 
This relations language extends our dynamical systems jargon. A homeomorphism $T$ on $X$ is a surjective relation on $X$ and a continuous surjection $F: X_{1} \rightarrow X$ is an action map between dynamical systems $\left(X_{1}, T_{1}\right)$ and $(X, T)$ exactly when it maps $T_{1}$ to $T$ as surjective relations. Motivated by this we will write $(W, R)$ for a finite set $W$ and a surjective relation $R$ on $W$.

For a relation $R$ on $W$ the reverse relation $R^{-1}={ }_{\text {def }}\{(b, a):(a, b) \in R\}$ is a surjective relation on $W$ when $R$ is. If $\phi$ maps $R_{1}$ to $R$, then it maps $R_{1}^{-1}$ to $R^{-1}$.

If $X$ is a Cantor space, $W$ is finite and $\phi: X \rightarrow W$ is a continuous surjective map, then the preimages of the points of $W$ form a decomposition $\mathcal{A}_{\phi}$ of $X$ consisting of nonempty clopen sets, hereafter just a decomposition. If $H$ is a homeomorphism on $X$, then $R=\phi \times \phi(H)$ is a surjective relation on $W$ and as described above we say that $\phi$ maps $H$ to $R$. We say that $H$ represents $R$ if some such continuous surjection $\phi$ exists.

If $\mathcal{A}$ is a decomposition of a Cantor space $X$ with metric $d$, then we define the mesh of $\mathcal{A}$, written $|\mathcal{A}|$, to be the maximum diameter of the elements of $\mathcal{A}$. For a map $\phi$ with domain $X$ we define the mesh of $\phi$, written $|\phi|$ to be the maximum diameter of the preimages of the points of the range of $\phi$. In particular, if $\phi$ is a continuous map with a finite range, then $|\phi|=\left|\mathcal{A}_{\phi}\right|$. Recall that a decomposition $\mathcal{A}_{1}$ refines a decomposition $\mathcal{A}_{2}$ if each element of $\mathcal{A}_{1}$ is contained in a, necessarily unique, element of $\mathcal{A}_{2}$. We collect a few standard facts.

Lemma 1.1. Let $X$ be a Cantor space with metric $d$.

(a) For a decomposition $\mathcal{A}_{1}$ of $X$ let $\epsilon>0$ be the minimum of the distances between any two distinct members of $\mathcal{A}_{1}$. Any decomposition $\mathcal{A}_{2}$ of $X$ with mesh less than $\epsilon$ refines $\mathcal{A}_{1}$.

(b) Let $\phi_{1}: X \rightarrow W_{1}$ and $\phi_{2}: X \rightarrow W_{2}$ be continuous surjections to finite sets. There exists $\rho: W_{2} \rightarrow W_{1}$ such that $\phi_{1}=\rho \circ \phi_{2}$ iff $\mathcal{A}_{\phi_{2}}$ refines $\mathcal{A}_{\phi_{1}}$. In that case the map $\rho$ is uniquely defined and is surjective.

(c) Let $\phi_{1}: X \rightarrow W_{1}$ be a continuous surjection with $W_{1}$ finite. There exists a positive number $\epsilon$ such that if $\phi_{2}: X \rightarrow W_{2}$ is any map with mesh less than $\epsilon$, then there exists a unique map $\rho: W_{2} \rightarrow W_{1}$ such that $\phi_{1}=\rho \circ \phi_{2}$.

Proof. In (a) it is clear that $\epsilon$ is a Lebesgue number for the open cover $\mathcal{A}_{1}$. Part (b) is an easy exercise and then (c) follows from (a) and (b).

To approximate the spiral, first we define the analogue of the spiral with periodic orbits at the ends instead of adding machines. For any nonnegative integer $n, \tilde{\Sigma}_{n}$ is the closed subset of $\mathbb{Z}_{*} \times \Theta_{n}$ ! given by

$$
\tilde{\Sigma}_{n} \quad=_{\text {def }} \quad\{(x, t): x= \pm \infty \quad \text { or } \quad x \in \mathbb{Z} \text { and } t=\Pi(x)\} .
$$

As before we let $\tau: \tilde{\Sigma}_{n} \rightarrow \tilde{\Sigma}_{n}$ denote the restriction of the product of the translation homeomorphisms. The projection $\pi: \Sigma \rightarrow \tilde{\Sigma}_{n}$ is the restriction of the product $1_{\mathbb{Z}_{*}} \times \pi$. It is an action map from $(\Sigma, \tau)$ to $\left(\tilde{\Sigma}_{n}, \tau\right)$. Since $n$ ! divides $(n+1)$ !, the projection $\pi$ factors to define projections $\pi:\left(\tilde{\Sigma}_{m}, \tau\right) \rightarrow\left(\tilde{\Sigma}_{n}, \tau\right)$ whenever $m \geq n$.

We obtain the finite set $\Sigma_{n}$ from $\tilde{\Sigma}_{n}$ by identifying the points $(x, t)$ and $(+\infty, t)$ for every $x \geq n$ and identifying the points $(x, t)$ and $(-\infty, t)$ for every $x \leq-n$. That is, the positive portion of the orbit beginning with $n$ is collapsed onto the periodic orbit at $+\infty$ while the negative portion up to $-n$ is collapsed onto the periodic orbit at $-\infty$. 
We let $\pi: \Sigma \rightarrow \Sigma_{n}$ and $\pi: \Sigma_{n} \rightarrow \Sigma_{n}$ denote the quotient maps. Let $R_{n} \subset$ $\Sigma_{n} \times \Sigma_{n}$ be the relation $\pi \times \pi(\tau)$ so that $\pi$ maps the homeomorphism $\tau$ to the surjective relation $R_{n}$ on $\Sigma_{n}$. The relation $R_{n}$ fails to be a bijective map only because the two points $(n-1, \Pi(n-1))$ and $(+\infty, \Pi(n-1))$ both relate via $R_{n}$ to $(n, \Pi(n))=(+\infty, \Pi(n))$ in $\Sigma_{n}$ and similarly $(-n+1, \Pi(-n+1))$ and $(-\infty, \Pi(-n+1))$ both relate via $R_{n}^{-1}$ to $(-n, \Pi(-n))=(-\infty, \Pi(-n))$ in $\Sigma_{n}$. If $m \geq n$, then the projection $\pi: \Sigma_{m} \rightarrow \Sigma_{n}$ maps $R_{m}$ to $R_{n}$.

The finite spiral $\Sigma_{0}$ consists of a single point, fixed by $R_{0}$. When $n>0$ then periodic orbits at the ends are recurrent points for the surjective relation $R_{n}$ and the remaining points, i.e. $\{(x, \Pi(x)):-n<x<n\}$ are the wandering points for $R_{n}$.

Lemma 1.2. (a) Let $\phi:(\Sigma, \tau) \rightarrow\left(\Sigma_{n}, R_{n}\right)$ be a continuous map of the spiral onto the finite spiral, i.e. $\phi$ maps $\tau$ to $R_{n}$. The preimage of each wandering point of the finite spiral is a single point of $\Sigma$ which is a wandering point of the spiral.

(b) Let $\phi:(\Sigma, \tau) \rightarrow\left(\Theta_{n}, \tau\right)$ be a continuous map of the spiral to the periodic orbit. The preimage of each point of $\Theta_{n}$ contains wandering points.

Proof. (a) It is easy to check that the image of a recurrent point is a recurrent point. Hence the preimage of a wandering point consists only of wandering points. If $x_{1}<x_{2}$ and $\phi\left(x_{1}, \Pi\left(x_{1}\right)\right)=\phi\left(x_{2}, \Pi\left(x_{2}\right)\right)$, then this image point is a periodic point for $R_{n}$.

(b) All of $\Theta_{n}$ is hit by any piece of length $n$ in any orbit sequence of $(\Sigma, \tau)$.

We will call $\left(\Sigma_{n}, R_{n}\right)$ a finite spiral of type $n$ and we will call the (wandering) point $(0, \Pi(0)) \in \Sigma_{n}$ the zero-point of the spiral.

Now we define the general construction of a space of spirals indexed by a pair $\left(A, A_{0}\right)$ where $A$ and $A_{0}$ are compact subsets of the unit interval $I$ such that

$$
A \supset A_{0} \supset \operatorname{Bdry} A,
$$

where $B d r y A$ is the topological boundary of $A$. Hence, $A \backslash A_{0}$ is an open subset of $\mathbb{R}$. It is the union of the countable set $\mathcal{J}\left(A \backslash A_{0}\right)$ of the disjoint open intervals which are the components of $A \backslash A_{0}$. If $j \in \mathcal{J}\left(A \backslash A_{0}\right)$, then $j=\left(j_{-}, j_{+}\right)$with endpoints $j_{-}, j_{+} \in A_{0}$.

Now assume, in addition, that $A_{0}$ is nowhere dense. We obtain the compact, zero-dimensional space $Z\left(A, A_{0}\right)$ from the disjoint union

$$
\mathcal{J}\left(A \backslash A_{0}\right) \times \Sigma \quad \cup \quad A_{0} \times \Theta
$$

by identifications so that in $Z\left(A, A_{0}\right)$,

$$
(j,-\infty, t)=\left(j_{-}, t\right) \quad \text { and } \quad(j,+\infty, t)=\left(j_{+}, t\right)
$$

for all $j \in \mathcal{J}\left(A \backslash A_{0}\right)$ and $t \in \Theta$. That is, after taking the product of $A$ with the group $\Theta$ we replace each interval $j \times \Theta$ by a copy of the spiral $\Sigma$. The homeomorphism $1_{\mathfrak{g}} \times \tau \cup 1_{A_{0}} \times \tau$ factors through the identifications to define the dynamical system $\left(Z\left(A, A_{0}\right), \tau_{\left(A, A_{0}\right)}\right)$. For each $r \in A_{0}$, the subset $\{r\} \times \Theta$ is an invariant set for $\tau_{\left(A, A_{0}\right)}$ on which $\tau_{\left(A, A_{0}\right)}$ is simply the adding machine translation $\tau$ on the $\Theta$ factor. For each $j \in \mathcal{J}\left(A \backslash A_{0}\right)$ the subset $\{j\} \times \Sigma$ is an invariant set for $\tau_{\left(A, A_{0}\right)}$ on which $\tau_{\left(A, A_{0}\right)}$ is the spiral $\tau$ on the $\Sigma$ factor. That is, we have a collection of adding machines indexed by the closed nowhere dense set $A_{0}$ with a countable number of gap pairs $j_{-}<j_{+}$of $A_{0}$ spanned by spirals. 
The space $Z\left(A, A_{0}\right)$ is compact and zero-dimensional, but the wandering points within the spirals are discrete. Denote by $C$ the classical Cantor set in the unit interval and define

$$
\begin{gathered}
X\left(A, A_{0}\right) \quad{ }_{\text {def }} Z\left(A, A_{0}\right) \times C, \\
T\left(A, A_{0}\right) \quad=_{\text {def }} \quad \tau_{\left(A, A_{0}\right)} \times 1_{C} .
\end{gathered}
$$

Thus, $T\left(A, A_{0}\right)$ is a homeomorphism on the Cantor space $X\left(A, A_{0}\right)$.

The projection map $A_{0} \times \Theta \rightarrow A_{0}$ which collapses each adding machine to a point extends to a continuous map $q: Z\left(A, A_{0}\right) \rightarrow A$ by embedding the orbit of wandering points of $\{j\} \times \Sigma$ in an order preserving manner to a bi-infinite sequence $\{q(j,(x, \Pi(x))): x \in \mathbb{Z}\}$ in the interval $j$ which converges to $j_{ \pm}$as $x \in \mathbb{Z}$ tends to $\pm \infty$.

Via $q$ we can pull back the ordering on $A \subset \mathbb{R}$ to obtain a total quasi-order on $Z\left(A, A_{0}\right)$.

On the other hand, the collapsing map $\zeta$ of (1.2) on each spiral defines

$$
\begin{aligned}
& \zeta: Z\left(A, A_{0}\right) \rightarrow \Theta \\
& \zeta(j,(x, t))=t \quad \text { for }(j,(x, t)) \in \mathcal{J}\left(A \backslash A_{0}\right) \times \Sigma, \\
& \zeta(a, t)=t \quad \text { for }(a, t) \in A_{0} \times \Theta .
\end{aligned}
$$

Clearly, $q \times \zeta: Z\left(A, A_{0}\right) \rightarrow I \times \Theta$ and $q \times \zeta \times \pi_{C}: X\left(A, A_{0}\right) \rightarrow I \times \Theta \times C$ are embeddings.

For $j \in \mathcal{J}\left(A \backslash A_{0}\right)$ we will call those spirals of $Z\left(A, A_{0}\right)$ or $X\left(A, A_{0}\right)$ which are mapped by $q$ into the closure $\bar{j}$ the spirals associated with $j$. So $\{j\} \times \Sigma$ is the unique spiral in $Z\left(A, A_{0}\right)$ associated with $j$ and the spirals in $X\left(A, A_{0}\right)$ associated with $j$ are of the form $\{j\} \times \Sigma \times\{c\}$ with $c \in C$.

We define certain canonical retractions from the spaces of spirals onto the individual spirals.

For each $j \in \mathcal{J}\left(A \backslash A_{0}\right)$ we define $r_{\left(A, A_{0}\right)}^{j}: Z\left(A, A_{0}\right) \rightarrow\{j\} \times \Sigma$. It collapses all the spirals left of $j$ to $\left\{j_{-}\right\} \times \Theta$ (which is identified with $\{j\} \times\{-\infty\} \times \Theta$ ), and all of the spirals right of $j$ to $\left\{j_{+}\right\} \times \Theta$. Finally, the spiral associated with $j$ is just fixed. In detail, with $\mathcal{J}=\mathcal{J}\left(A \backslash A_{0}\right)$,

$$
\begin{gathered}
r_{\left(A, A_{0}\right)}^{j} Z\left(A, A_{0}\right) \rightarrow\{j\} \times \Sigma \quad \subset \quad Z\left(A, A_{0}\right) \\
r_{\left(A, A_{0}\right)}^{j}(a, t)= \begin{cases}\left(j_{-}, t\right) & \text { for }(a, t) \in A_{0} \times \Theta \text { with } a \leq j_{-} \\
\left(j_{+}, t\right) & \text { for }(a, t) \in A_{0} \times \Theta \text { with } a \geq j_{+}\end{cases} \\
r_{\left(A, A_{0}\right)}^{j}(z,(x, t))= \begin{cases}\left(j_{-}, t\right) & \text { for }(z,(x, t)) \in \mathcal{J} \times \Sigma \text { with } z_{+} \leq j_{-} \\
\left(j_{+}, t\right) & \text { for }(z,(x, t)) \in \mathcal{J} \times \Sigma \text { with } z \geq j_{+} \\
(j,(x, t)) & \text { for }(x, t) \in \Sigma \text { with } z=j .\end{cases}
\end{gathered}
$$

If $c \in C$, then define

$$
r_{\left(A, A_{0}\right)}^{j c}: X\left(A, A_{0}\right) \rightarrow\{j\} \times \Sigma \times\{c\}
$$

by projecting first from $X\left(A, A_{0}\right)=Z\left(A, A_{0}\right) \times C$ to $Z\left(A, A_{0}\right) \times\{c\}$ and then using $r_{\left(A, A_{0}\right)}^{j}$ on the $Z\left(A, A_{0}\right)$ factor. Clearly, $r_{\left(A, A_{0}\right)}^{j c}$ maps $T\left(A, A_{0}\right)$ to its restriction on the single spiral.

Examples. Let $I$ be the unit interval with boundary $\dot{I}=\{0,1\}$ and $C$ be the classical Cantor set in $I$ consisting of those points $a$ which admit a ternary expansion 
. $a_{0} a_{1} a_{2} \ldots$ with no $a_{i}=2$. Let $D$ consist of those points $a$ which admit a ternary expansion.$a_{0} a_{1} a_{2} \ldots$ such that the smallest index $i=0,1, \ldots$ with $a_{i}=2$ - if any $a_{i}$ does equal 2 - is even. That is, for the Cantor set $C$ we eliminate all the middle third open intervals, first one of length $1 / 3$, then two of length $1 / 9$, then four of length $1 / 27$ and so forth. For $D$ we retain the interval of length $1 / 3$, eliminate the two of length $1 / 9$, keep the four of length $1 / 27$, eliminate the eight of length $1 / 81$ and so forth. The boundary of $D$ is the Cantor set $C . \mathcal{J}(D \backslash C)$ consists of the open intervals of length $1 / 3^{2 k+1}$ which we retained in $D$ whereas $\mathcal{J}(I \backslash D)$ consists of the open intervals of length $1 / 3^{2 k}$ which we eliminated from $D$.

Notice that between any two subintervals in $\mathcal{J}(D \backslash C) \cup \mathcal{J}(I \backslash D)=\mathcal{J}(I \backslash C)$ there occur infinitely many intervals of $\mathcal{J}(D \backslash C)$ and of $\mathcal{J}(I \backslash D)$. We let $[D]$ denote the set of components of $D$. A component of $D$ is either a closed interval $\bar{j}$ for $j \in \mathcal{J}(D \backslash C)$ or a point $a$ of $C$ which is not the endpoint of an interval in $\mathcal{J}(D \backslash C)$.

- $\left(A, A_{0}\right)=(I, \dot{I}): \mathcal{J}(I \backslash \dot{I})$ consists of the single open interval $(0,1)$ and so $\left(Z(I,\{0,1\}),, \tau_{(I, \dot{I})}\right)$ is just a single spiral. The homeomorphism $T(I, \dot{I})$ is a product of spirals indexed by the Cantor set $C$.

- $\left(A, A_{0}\right)=(I, C)$ : We call $\left(Z(I, C), \tau_{(I, C)}\right)$ a line of spirals. Let $x_{0}, x_{1} \in$ $Z(I, C)$ with $q\left(x_{0}\right) \leq q\left(x_{1}\right)$ (recall from above the map $q: Z(I, C) \rightarrow I$ obtained by collapsing the adding machines and embedding the spirals). For every $\epsilon>0$ there is an $\epsilon$ chain from $x_{0}$ to $x_{1}$. That is, the chain relation $\mathfrak{e}_{(I, C)}$ on $Z(I, C)$ is exactly the total quasi-order induced by $q$ from $\mathbb{R}$.

- $\left(A, A_{0}\right)=(D, C)$ : We call $\left(Z(D, C), \tau_{(I, C)}\right)$ a Cantor set of spirals. Of course, there are only countably many spirals, and the ordering on the set of spirals is order dense just as in the previous example. However, this time $q: Z(D, C) \rightarrow D$ induces a much larger order than the chain order $\mathrm{C}_{\tau_{(D, C)}}$. If $x_{0}$ is on a spiral and $x_{1}$ is not on the same spiral, then $q\left(x_{0}\right)$ and $q\left(x_{1}\right)$ are separated by a gap in $I \backslash D$ of length greater than $\epsilon$ provided $\epsilon$ is sufficiently small. This gap cannot be crossed by an $\epsilon$ chain for $\tau_{(D, C)}$. It follows that this time the chain relation $\mathrm{C} \tau_{(D, C)}$ is exactly the orbit closure relation for $\tau_{(D, C)}$. We will call $T(D, C)$ the Special Homeomorphism of the Cantor Space $X(D, C)$.

\section{The LifTing LEMMA}

Our main tool will be a result which characterizes the Special Homeomorphism $T(D, C): X(D, C) \rightarrow X(D, C)$.

From a spiral $\Sigma$ to a finite spiral $\Sigma_{n}$ there are three maps which we will need: $\xi_{L}$ collapses $\Sigma$ to $\Theta$ via $\zeta$ of (1.2), then projects $\Theta$ to the cyclic group $\Theta_{n \text { ! }}$ and then identifies $\Theta_{n}$ ! with $\{-\infty\} \times \Theta_{n !} \subset \Sigma_{n}$. Similarly, $\xi_{R}$ collapses and projects and then identifies with $\{+\infty\} \times \Theta_{n !}$. We use $\xi_{M}$ as a new label for the canonical projection $\pi: \Sigma \rightarrow \Sigma_{n}$. When $m>n$ each of these factors is used to define a map from $\Sigma_{m}$ into $\Sigma_{n}$ : the canonical projection $\xi_{M}$, and $\xi_{L}$ and $\xi_{R}$ which collapse onto the cycles at the left and right ends, respectively. When $n=0$ these three maps are all the unique map to the singleton set $\Sigma_{0}$.

We now build an inverse system of relations on finite sets which will have a limit isomorphic to the Special Homeomorphism $T(D, C)$.

Begin with a six symbol alphabet $\left\{L_{1}, L_{2}, M_{1}, M_{2}, R_{1}, R_{2}\right\}$. For $n=0,1,2, \ldots$ let $B_{n}$ denote the set of words of length $n$ on this alphabet. Thus, for example, $B_{0}$ 
consists of the single empty word $\emptyset$ of length 0 . Let

$$
\mathcal{W}_{n} \quad=_{\text {def }} \quad B_{n} \times \Sigma_{n} \quad \text { for } n=0,1, \ldots
$$

That is, $\mathcal{W}_{n}$ is a list of finite spirals of type $n$, indexed by words of length $n$. We define the relation $R_{n}$ on $\mathcal{W}_{n}$ by using the relation $R_{n}$ on each finite spiral $\Sigma_{n}$.

We will label separately the three $R_{n}$ invariant subsets of the spiral $\{\omega\} \times \Sigma_{n}$ corresponding to the word $\omega$ and call these the pieces of $\{\omega\} \times \Sigma_{n}$ :

$$
\begin{aligned}
& S^{\omega}={ }_{\text {def }} \quad\{\omega\} \times \Sigma_{n}, \\
& \quad G_{ \pm}^{\omega}={ }_{\text {def }} \quad\{\omega\} \times\{ \pm \infty\} \times \Theta_{n !} .
\end{aligned}
$$

That is, $S^{\omega}$ is the entire finite spiral while $G_{-}^{\omega}$ and $G_{+}^{\omega}$ are the periodic orbits on the left and right ends of the spiral.

Now suppose that $\omega$ is a word of length $n$ and that $K_{\alpha}$ is a letter of the alphabet ( $K=L, M$ or $R$ and $\alpha=1$ or 2 ) so that $\omega K_{\alpha} \in B_{n+1}$. Define

$$
\xi:\left\{\omega K_{\alpha}\right\} \times \Sigma_{n+1} \rightarrow\{\omega\} \times \Sigma_{n} \quad \text { by } \xi=\xi_{K} .
$$

That is, when $K=L$ we use the left collapse mapping onto $G_{-}^{\omega}$, when $K=R$ we use the right collapse onto $G_{+}^{\omega}$, and when $K=M$ we use the projection onto $S^{\omega}$. Concatenate these maps to define $\xi: \mathcal{W}_{n+1} \rightarrow \mathcal{W}_{n}$. Clearly, $\xi$ maps $R_{n+1}$ to $R_{n}$.

Moving from $\mathcal{W}_{n}$ to $\mathcal{W}_{n+1}$ each cyclic end is unwrapped from period $n$ ! to period $(n+1)$ ! and the finite spirals between are extended from length $2 n+1$ to length $2 n+3$. Each spiral of type $n$ is hit via $\xi$ by six spirals of type $n+1$, two hit each end and two project onto the entire spiral.

For any positive integer $k$ we will write $\xi: \mathcal{W}_{n+k} \rightarrow \mathcal{W}_{n}$ for the composition of the projections $\mathcal{W}_{n+k} \rightarrow \mathcal{W}_{n+k-1} \rightarrow \ldots \rightarrow \mathcal{W}_{n+1} \rightarrow \mathcal{W}_{n}$. Notice that each piece of a spiral in $\mathcal{W}_{n}$ is the $\xi$ image of $2 \cdot 6^{k-1}$ spirals in $\mathcal{W}_{n+k}$.

We let $\mathcal{W}_{\infty}$ denote the inverse limit of this sequence of spaces and let $R_{\infty} \subset$ $\mathcal{W}_{\infty} \times \mathcal{W}_{\infty}$ denote the inverse limit of the $R_{n}$ 's. It is clear that $\mathcal{W}_{\infty}$ is a compact, zero-dimensional space, and it is easy to check that $R_{\infty}$ is a closed surjective relation on it.

We will now see that $\left(\mathcal{W}_{\infty}, R_{\infty}\right)$ is isomorphic to $(X(D, C), T(D, C))$. If we had used the three element alphabet $\{L, M, R\}$ we would have instead obtained a copy of $\left(Z(D, C), \tau_{(D, C)}\right)$. The doubling at each stage produces the product with the extra Cantor space factor $C$.

Definition 2.1. Let $T: X \rightarrow X$ be a homeomorphism of a Cantor space equipped with a metric $d$. We say that $T$ satisfies the Lifting Property for the inverse system $\left\{\mathcal{W}_{i} ; \xi\right\}$ described above when the following condition holds.

Whenever $\phi: X \rightarrow \mathcal{W}_{n}$ is a continuous surjection which maps $(X, T)$ to $\left(\mathcal{W}_{n}, R_{n}\right)$, and $\epsilon$ is a positive real number, there exists, for some positive integer $k$, a continuous surjection $\rho: X \rightarrow \mathcal{W}_{n+k}$ which maps $(X, T)$ to $\left(\mathcal{W}_{n+k}, R_{n+k}\right)$ such that the mesh of $\rho$ is at most $\epsilon$ and, in addition, $\phi=\xi \circ \rho$ where $\xi: \mathcal{W}_{n+k} \rightarrow \mathcal{W}_{n}$ is the projection in the inverse system.

Since all metrics on a Cantor space are uniformly equivalent, the Lifting Property for $(X, T)$ is independent of the choice of metric.

Lemma 2.2 (The Lifting Lemma). The Special Homeomorphism $T(D, C)$ : $X(D, C) \rightarrow X(D, C)$ satisfies the Lifting Property. 
Proof. For the duration of the proof we drop the labels associated with $(D, C)$, writing $X$ for $X(D, C), Z$ for $Z(D, C)$, and $\mathcal{J}$ for $\mathcal{J}(D \backslash C)$. Recall that we write $[D]$ for the set of components of $D$. Such a component is either the closure $\bar{j}$ of an interval $j \in \mathcal{J}$ or a point $a \in C$ which is not an endpoint of such an interval.

Since all metrics on $X$ are uniformly equivalent we can choose any one which is convenient to work with. On the unit interval $I$ and so on the Cantor set $C$ use the metric induced from $\mathbb{R}$. On the compact group $\Theta$ choose a translation invariant metric with diameter 1 . On the product $I \times \Theta \times C$ use the max metric given by these three on the separate coordinates. Finally, let $d$ be the metric on $X$ which is pulled back from this one via the embedding $q \times \zeta \times \pi_{C}$. Hence, $Y \subset X$ has diameter less than $\epsilon$ iff $q(Y), \pi_{C}(Y) \subset I$ and $\zeta(Y) \subset \Theta$ all have diameter less than $\epsilon$.

We are given a continuous surjection $\phi:(X, T) \rightarrow\left(\mathcal{W}_{n}, R_{n}\right)$ for which we must construct a suitable lift.

By shrinking $\epsilon$ we can (and do) assume that $\epsilon$ is smaller than the distance between any two distinct elements of the decomposition $\mathcal{A}_{\phi}$ induced by $\phi$. Hence, $\phi$ is constant on any subset of $X$ with diameter $\epsilon$ or less.

Choose a decomposition $\mathcal{V}$ of $C$ with mesh less than $\epsilon$.

The kernels of the homomorphisms $\pi: \Theta \rightarrow \Theta_{(n+k)}$ ! form a decreasing sequence of compact subgroups with intersection $\{0\}$. Hence, there exists a positive integer $k_{1}$ so that the diameter of the kernel is less than $\epsilon$ when $k \geq k_{1}$. Hence, when $k \geq k_{1}$ the mesh of $\pi$ is less than $\epsilon$.

Since the intervals of $\mathcal{J}$ are disjoint and are contained in $I$, only finitely many members of $\mathcal{J}$ have length $\epsilon / 2$ or more. We will call these the large intervals in $\mathcal{J}$. We will denote by $\mathcal{J}_{\text {large }}$ the finite set of large intervals.

For each $j \in \mathcal{J}$ the wandering points of the corresponding spiral are mapped by $q$ to a bi-infinite convergent sequence. That is, $\{q(j,(x, \Pi(x))): x \in \mathbb{Z}\}$ converges to $j_{ \pm}$as $x$ tends to $\pm \infty$. Hence, we can choose a positive integer $k_{2}$ so that for every $j \in \mathcal{J}_{\text {large }}\left|j_{+}-q\left(j,\left(n+k_{2}, \Pi\left(n+k_{2}\right)\right)\right)\right|<\epsilon / 2$ and $\left|j_{-}-q\left(j,\left(-n-k_{2}, \Pi\left(-n-k_{2}\right)\right)\right)\right|<\epsilon / 2$ and so the sets $\{q(j,(x, \Pi(x))): x \geq$ $\left.n+k_{2}\right\} \cup\left\{j_{+}\right\}$and $\left\{q(j,(x, \Pi(x))): x \leq-n-k_{2}\right\} \cup\left\{j_{-}\right\}$have diameter less than $\epsilon / 2$. Notice that for the remaining intervals $j$ the entire sequence has diameter less than $\epsilon / 2$.

Now we consider $\phi$. It maps onto $\mathcal{W}_{n}$ which consists of finite spirals indexed by the set $B_{n}$ of words of length $n$. For each word $\omega \in B_{n}$ there is a finite spiral, $S^{\omega}$. At the left and right ends $S^{\omega}$ contains the cycles of period $n$ ! labeled $G_{-}^{\omega}$ and $G_{+}^{\omega}$. Since $\phi$ maps the homeomorphism $T$ to $R_{n}$ the image under $\phi$ of any spiral must be either some $S^{\omega}$ or some $G_{ \pm}^{\omega}$. We will call the spirals which map onto some $S^{\omega}$ central spirals and the others end spirals. The image under $\phi$ of any adding machine contains only recurrent points and so must be some $G_{ \pm}^{\omega}$.

Each $\phi^{-1}\left(S^{\omega}\right)$ is a clopen invariant subset of $X$ and each $\phi^{-1}\left(G_{ \pm}^{\omega}\right)$ is a closed invariant subset of $X$. Since $\phi$ is surjective all of these sets are nonempty. Each is a union of elements of $\mathcal{A}_{\phi}$. In particular, the distance between $\phi^{-1}\left(G_{-}^{\omega}\right)$ and $\phi^{-1}\left(G_{+}^{\omega}\right)$ is larger than $\epsilon$.

It follows that any spiral associated with an interval $j \in \mathcal{J}$ of length less than $\epsilon$ is an end spiral. To see this note that for any $t \in \Theta$ and $c \in C$ the distance between the points $(j,(-\infty, t), c)=\left(j_{-}, t, c\right)$ and $(j,(+\infty, t), c)=\left(j_{+}, t, c\right)$ is exactly the length of $j$. These points lie at opposite ends of the spiral. Hence, if the spiral 
maps onto $S^{\omega}$, then these points map into $G_{-}^{\omega}$ and $G_{+}^{\omega}$ respectively. So this can't happen when the length of $j$ is less than $\epsilon$. It follows that central spirals are associated only with large intervals.

Now for every interval $j \in \mathcal{J}$ its closure is a component of $D$ and so we can choose a clopen subset $U_{j}$ of $D$ which contains $\bar{j}$ and which is arbitrarily close to $\bar{j}$.

For each $j \in \mathcal{J}_{\text {large }}$ we choose a clopen $U_{j}$ containing $\bar{j}$ so that the following conditions are satisfied;

(1) $U_{j} \subset\left(j_{-}-\epsilon / 2, j_{+}+\epsilon / 2\right)$.

(2) If $j_{1}, j_{2} \in J_{\text {large }}$ with $j_{1} \neq j_{2}$, then $U_{j_{1}}$ and $U_{j_{2}}$ are disjoint.

(3) For each $j \in \mathcal{J}_{\text {large }}$ the sets

$$
\left(j_{-}-\epsilon / 2, j_{-}\right) \cap\left(D \backslash U_{\text {large }}\right) \quad \text { and } \quad\left(j_{+}, j_{+}+\epsilon / 2\right) \cap\left(D \backslash U_{\text {large }}\right)
$$

are nonempty where

$$
U_{\text {large }}=\bigcup\left\{U_{j}: j \in \mathcal{J}_{\text {large }}\right\} .
$$

Note that the intervals of $\mathcal{J}$ have pairwise disjoint closures and there are only finitely many large intervals. For the last condition observe that the singleton components are dense in $C$ (they are the complement of the countable set of endpoints of the intervals in $\mathcal{J}$ ) and so they occur arbitrarily close to each endpoint of any interval $j$. Thus by shrinking the $U_{j}$ 's if necessary we can make sure that the required outside points exist.

The components of $D$ which are not contained in the clopen set $U_{\text {large }}$ all have diameter less than $\epsilon / 2$. Hence, we can choose a collection $\tilde{\mathcal{U}}$ of nonempty clopen subsets each of diameter less than $\epsilon / 2$ such that

$$
\mathcal{U}==_{\text {def }} \quad\left\{U_{j}: j \in \mathcal{J}_{\text {large }}\right\} \cup \tilde{\mathcal{U}}
$$

is a decomposition of $D$.

Consider $U$ any nonempty clopen subset of $D$ and $V$ any nonempty clopen subset of $C$. The clopen subset $q^{-1}(U) \times V$ of $X$ is invariant with respect to $T$. Because $U$ is clopen it contains any intervals of $\mathcal{J}$ which meet it. Since the union of these intervals is dense in $D$ it follows that $U$ contains some such interval $j$. With $c \in V$ consider the restriction to $q^{-1}(U) \times V$ of the retraction $r^{j c}: X \rightarrow\{j\} \times \Sigma \times\{c\}=q^{-1}(\bar{j}) \times\{c\}$ defined in (1.9) and (1.10).

Recall that the metric on $X$ is given by the embedding $q \times \zeta \times \pi_{C}$. Assume that $V$ has diameter less than $\epsilon$ and that either the diameter of $U$ is less than $\epsilon / 2$ or $j \in \mathcal{J}_{\text {large }}$ and $U=U_{j}$. The collapse from $V$ to $c$ moves the $C$ coordinate given by $\pi_{C}$ a distance less than $\epsilon$. The $\Theta$ coordinate given by $\zeta$ is not moved at all. By condition (1) on $U_{j}$ it follows that in either case the $D$ coordinate given by $q$ is moved a distance less than $\epsilon / 2$.

Since $\phi$ is constant on sets of diameter less than $\epsilon$ we have that

$$
\phi \circ r^{j c}\left|\left(q^{-1}(U) \times V\right)=\phi\right|\left(q^{-1}(U) \times V\right) .
$$

In particular, $\phi$ maps the entire clopen set $q^{-1}(U) \times V$ to the image of the single spiral $\{j\} \times \Sigma \times\{c\}=q^{-1}(\bar{j}) \times\{c\}$.

$$
\tilde{\mathcal{Q}}={ }_{\text {def }} \quad\left\{q^{-1}(U) \times V: U \in \mathcal{U} \text { and } V \in \mathcal{V}\right\}
$$

is an invariant decomposition of $X$ on each element of which $\phi$ maps onto $S^{\omega}, G_{-}^{\omega}$ or $G_{+}^{\omega}$ for some word $\omega \in B_{n}$. 
Furthermore, for each word $\omega$ each of these three pieces is the image of some member of $\tilde{\mathcal{Q}}$. Since $\phi$ is onto, $S^{\omega}$ must be the image of some spiral and such a central spiral is associated with a large interval $j$. So for some $V \in \mathcal{V}, \phi$ maps $q^{-1}\left(U_{j}\right) \times V$ to $S^{\omega}$. By condition (3) there exist $U_{-}, U_{+} \in \tilde{\mathcal{U}}$ such that $U_{-} \cap\left(j_{-}-\epsilon / 2, j_{-}\right) \neq \emptyset$ and $U_{+} \cap\left(j_{+}, j_{+}+\epsilon / 2\right) \neq \emptyset$. For the same $V, \phi$ maps $q^{-1}\left(U_{-}\right) \times V$ to $G_{-}^{\omega}$ because for $c \in V$ all of the points of $q^{-1}\left(U_{-}\right) \times V$ are within $\epsilon$ of the points in the adding machine $\left\{j_{-}\right\} \times \Theta \times\{c\}$ which is mapped by $\phi$ to $G_{-}^{\omega}$. Similarly, $q^{-1}\left(U_{+}\right) \times V$ maps to $G_{+}^{\omega}$.

Now let $M$ be the maximum number of elements of $\tilde{Q}$ which are mapped onto any $S^{\omega}$ or $G_{ \pm}^{\omega}$ piece as $\omega$ varies over $B_{n}$.

At last, we choose $k_{0}>\max \left(k_{1}, k_{2}\right)$ and such that $2 \cdot 6^{k_{0}-1} \geq M$. Let $k$ be any integer greater than or equal to $k_{0}$. We can refine $\tilde{\mathcal{Q}}$ to obtain a clopen decomposition $\mathcal{Q}$ each element of which is of the form $q^{-1}(U) \times V_{1}$ with $U \in \mathcal{U}$ and $V_{1}$ a clopen subset of some element $V$ of $\mathcal{V}$, and, furthermore, each $S^{\omega}$ and each $G_{ \pm}^{\omega}$ is hit by exactly $2 \cdot 6^{k-1}$ elements of $\mathcal{Q}$.

To see this observe that if $q^{-1}(U) \times V$ hits some piece we can decompose $V$ into clopen sets $V_{1}, V_{2}, \ldots$ to increase the number of hits as much as we need. Here we use that every piece is hit at least once.

We can therefore choose a bijection $\tilde{\rho}: \mathcal{Q} \rightarrow \mathcal{W}_{n+k}$ such that for every $Q \in \mathcal{Q}$, $\tilde{\rho}(Q)$ is a finite spiral in $\mathcal{W}_{n+k}$ which projects to the same piece $S^{\omega}$ or $G_{ \pm}^{\omega}$ via $\xi$ as $Q$ is mapped by $\phi$.

For each $Q=q^{-1}(U) \times V_{1}$ we choose an interval from $\mathcal{J}$ which is contained in $U$. If $U=U_{j}$ with $j \in \mathcal{J}_{\text {large }}$, then we make $j$ the choice. Choose $c \in V_{1}$ and let $r^{j c}$ denote the restriction to $Q$ of the retraction onto the spiral $q^{-1}(\bar{j}) \times\{c\}$. $\phi$ maps this spiral, and hence all of $Q$, onto a piece of the form $S^{\omega}$ or $G_{ \pm}^{\omega}$ for some word $\omega \in B_{n} . \tilde{\rho}(Q)=W$ is a finite spiral in $\mathcal{W}_{n+k}$ which is mapped by $\xi$ onto the same piece.

Now let $z$ be the zero-point of $W$. If $\xi(W)$ is a central piece and so $q^{-1}(\bar{j}) \times\{c\}$ is a central spiral, then $\xi(z)$ is the zero-point of this central piece. Otherwise, $\xi(z)$ is just some point of one of the periodic orbits $G_{ \pm}^{\omega}$ which is the $\phi$ image of the end spiral $q^{-1}(\bar{j}) \times\{c\}$. In either case, Lemma 1.2 shows that there exists a wandering point $(j,(x, \Pi(x)), c)$ such that $\phi(j,(x, \Pi(x)), c)=\xi(z)$. Let $\rho(j,(x, \Pi(x)), c)=z$. There is a unique map $\rho: q^{-1}(\bar{j}) \times\{c\} \rightarrow W$ which satisfies this condition and which maps $T$ to $R_{n+k}$ on $W$. Then extend by using the retraction $r^{j c}$, i.e. define $\rho \circ r^{j c}: Q \rightarrow W$.

The concatenation of these maps as $Q$ varies over $\mathcal{Q}$ is our required $\rho:(X, T) \rightarrow$ $\left(\mathcal{W}_{n+k}, R_{n+k}\right)$.

It follows from (2.6) that the map $\rho: X \rightarrow \mathcal{W}_{n}$ satisfies $\phi=\xi \circ \rho$. To check that the mesh is at most $\epsilon$ we have to worry about the identifications made in projecting from a spiral in $X$ to a finite spiral in $\mathcal{W}_{n+k}$. The identifications of $\Theta$ coordinates occur across distances less than $\epsilon$ because $k>k_{1}$. The identifications of $D$ coordinates occur between points of distance less than $\epsilon$ because $k>k_{2}$. See also condition (1). Since distinct elements of $\mathcal{Q}$ map to distinct spirals in $\mathcal{W}_{n+k}$ the mesh of $\rho$ is less than $\epsilon$.

Corollary 2.3. A homeomorphism $(X, T)$ satisfies the Lifting Property iff it is isomorphic to the Special Homeomorphism $(X(D, C), T(D, C))$, i.e. there exists a homeomorphism $H: X \rightarrow X(D, C)$ such that $T=H \circ T(D, C) \circ H^{-1}$. 
Proof. It is easy to see that the Lifting Property is an isomorphism invariant. That is, if $H: X_{1} \rightarrow X$ is a homeomorphism mapping $T_{1}$ on $X_{1}$ to $T$ on $X$, then $\left(X_{1}, T_{1}\right)$ satisfies the Lifting Property iff $(X, T)$ does. So the Lifting Lemma implies that any isomorph of $(X(D, C), T(D, C))$ satisfies the Lifting Property.

Assume that $(X, T)$ satisfies the Lifting Property. Fix a metric $d$ on $X$ and fix a decreasing, positive sequence $\left\{\epsilon_{i}: i=1,2, \ldots\right\}$ converging to zero. Let $\phi_{0}$ be the map from $X$ to the singleton space $\mathcal{W}_{0}$ which maps $T$ to $R_{0}$. Let $n_{0}=0$.

We construct a sequence of maps $\phi_{i}: X \rightarrow \mathcal{W}_{n_{i}}$ with mesh less than $\epsilon_{i}$ which map $T$ to $R_{n_{i}}$ and such that $\phi_{i}=\xi \circ \phi_{i+1}$ where $\xi$ is the projection from $\mathcal{W}_{n_{i+1}}$ to $\mathcal{W}_{n_{i}}$. This is an inductive construction where we use the Lifting Property to go from $\phi_{i}$ to $\phi_{i+1}$ with $n_{i+1}=n_{i}+k$ for suitable positive $k$.

Since the increasing sequence $\left\{n_{i}\right\}$ is cofinal in the positive integers we obtain a continuous surjection $\phi_{\infty}$ from $X$ to the inverse limit space $\mathcal{W}_{\infty}$. Furthermore, the continuous map $\phi_{\infty}$ maps $T$ onto the limit relation $R_{\infty}$. But because the mesh of the $\phi_{i}$ 's tends to zero, it follows that $\phi_{\infty}$ is a homeomorphism. Since $\phi$ maps $T$ onto $R_{\infty}$ it follows that the latter is the homeomorphism $\phi_{\infty} \circ T \circ \phi_{\infty}^{-1}$.

That is, we have shown that $R_{\infty}$ is a homeomorphism on the compact space $\mathcal{W}_{\infty}$ and that $(X, T)$ is isomorphic to $\left(\mathcal{W}_{\infty}, R_{\infty}\right)$. In particular, $(X(D, C), T(D, C))$ is isomorphic to $\left(\mathcal{W}_{\infty}, R_{\infty}\right)$ and so $(X, T)$ is isomorphic to $(X(D, C), T(D, C))$ as required. It follows from this construction that $\mathcal{W}_{\infty}$ is a Cantor space and $R_{\infty}$ is a homeomorphism on it.

We will call a homeomorphism $T: X \rightarrow X$ a Special Homeomorphism on $X$ when $(X, T)$ is isomorphic to the canonical example $(X(D, C), T(D, C))$. Since all Cantor spaces are homeomorphic, all admit Special Homeomorphisms.

\section{The SPECIAL HOMEOMORPHISMS ARE GENERIC}

For a compact metrizable space $X$, let $\mathcal{H}(X)$ denote the homeomorphism group for $X$. Equipped with the topology of uniform convergence it is a Polish topological group. We consider the adjoint action of this group on itself and for $T \in \mathcal{H}(X)$ we let $\mathcal{O}(T)$ denote the orbit of $T$ with respect to this action. Thus,

$$
\mathcal{O}(T) \quad=_{\text {def }} \quad\left\{H \circ T \circ H^{-1}: H \in \mathcal{H}(X)\right\} .
$$

That is, $T_{1} \in \mathcal{O}(T)$ exactly when $\left(X, T_{1}\right)$ is isomorphic to $(X, T)$. For example, if $X$ is a Cantor space, then the set of Special Homeomorphisms on $X$, which we will denote as $\mathcal{S}_{X}$, is a single orbit.

We call a homeomorphism $T$ of dense type when its orbit is dense in $\mathcal{H}(X)$, i.e. when $T$ is in the set

$$
\mathcal{T}_{X} \quad=_{\text {def }} \quad\{T \in \mathcal{H}(X): \overline{\mathcal{O}(T)}=\mathcal{H}(X)\} .
$$

Thus, $T$ is of dense type when every homeomorphism on $X$ can be uniformly approximated, arbitrarily closely, by a homeomorphism isomorphic with $T$. We say that $X$ satisfies the Rohlin Property when it admits a homeomorphism of dense type, or, equivalently, when $\mathcal{T}_{X} \neq \emptyset$.

Of course, the Rohlin Property for $X$ just says that the adjoint action of $\mathcal{H}(X)$ on itself is topologically transitive and $\mathcal{T}_{X}$ is exactly the set of transitive points for this action. We use this alternative language to avoid confusion with the situation that the dynamical system $(X, T)$ is topologically transitive, i.e. that the $\mathbb{Z}$ action on $X$ via $T$ is topologically transitive. 
Recall from the previous section that if $R$ is a surjective relation on a finite set $W$, then a continuous map $\phi: X \rightarrow W$ maps $T \in \mathcal{H}(X)$ to $R$ when $(\phi \times \phi)(T)=R$. This says exactly that for all $a, b \in W$,

$$
(a, b) \in R \quad \Longleftrightarrow \quad T\left(\phi^{-1}(a)\right) \cap \phi^{-1}(b) \neq \emptyset .
$$

This is a clopen condition on $T$ and so

$$
\mathcal{H}[\phi, R] \quad=_{\text {def }} \quad\{T \in \mathcal{H}(X):(\phi \times \phi)(T)=R\}
$$

is a clopen subset of $\mathcal{H}(X)$. We say that $T$ represents $R$ when some such continuous map exists. The set of homeomorphisms which represent $R$,

$$
\mathcal{H}[X, R] \quad=_{\text {def }} \bigcup_{\phi} \mathcal{H}[\phi, R],
$$

is an open subset of $\mathcal{H}(X)$ where the union is taken over all continuous maps $\phi: X \rightarrow W$.

That the Cantor space satisfies the Rohlin Property was proved by Glasner and Weiss GW and by Akin, Hurley and Kennedy AHK.

We quote the relevant results from the latter.

Theorem 3.1. Let $X, X_{1}$ be Cantor spaces.

(a) For any surjective relation $R$ on a finite set $W$ the open set $\mathcal{H}[X, R]$ is dense in $\mathcal{H}(X)$. In addition, it is invariant with respect to the adjoint action. In fact, if $T \in \mathcal{H}[X, R], T_{1} \in \mathcal{H}\left(X_{1}\right)$, and there exists $H: X_{1} \rightarrow X$ a continuous surjection which maps $T_{1}$ to $T$, i.e. $H \circ T_{1}=T \circ H$, then $T_{1} \in \mathcal{H}\left[X_{1}, R\right]$.

(b) X satisfies the Rohlin Property with

$$
\mathcal{T}_{X}=\bigcap_{F} \mathcal{H}[X, R]
$$

where the intersection is taken over all surjective relations $F$ defined on some finite subset of a fixed countable set.

(c) If $T \in \mathcal{T}_{X}, T_{1} \in \mathcal{H}\left(X_{1}\right)$, and there exists $H: X_{1} \rightarrow X$ a continuous surjection which maps $T_{1}$ to $T$, i.e. $H \circ T_{1}=T \circ H$, then $T_{1} \in \mathcal{T}_{X_{1}}$.

Proof. (a) The density of $\mathcal{H}[X, R]$ is Theorem 8.3 of Akin, Kennedy and Hurley AHK. Clearly, if $\phi$ maps $T$ to $R$ and $H$ maps $T_{1}$ to $T$, then $\phi \circ H$ maps $T_{1}$ to $R$.

(b) The characterization of $\mathcal{T}_{X}$ in (3.6) is Theorem 8.4 of Akin, Kennedy and Hurley AHK.

Notice that one direction is easy because the set of transitive points for any action is contained in any nonempty open subset which is invariant with respect to the action.

From the Baire Category Theorem it follows that $\mathcal{T}_{X}$ is a dense $G_{\delta}$ set and so is nonempty. This is an example of the Oxtoby Philosophy repeatedly displayed in Oxtoby [0]: to prove that a set is nonempty it is often easiest to prove that the set is residual.

(c) This is immediate from (a) and (b).

Thus, a homeomorphism on a Cantor space is of dense type when it represents every surjective relation on any finite set. This is an easy characterization to use. 
Proposition 3.2. If $A$ is a closed subset of the unit interval $I$ and $A_{0}$ is a closed, nowhere dense, proper subset of $A$ which contains BdryA, then the homeomorphism $T\left(A, A_{0}\right)$ is of dense type in $\mathcal{H}\left(X\left(A, A_{0}\right)\right)$.

Proof. Since $A_{0}$ is a proper subset of $A$, the set $\mathcal{J}\left(A \backslash A_{0}\right)$ is nonempty. If $j$ is any one of these intervals, then we can retract $A$ onto $\bar{j}$ and retract $Z\left(A, A_{0}\right)$ onto the spiral associated with $j$ as in (1.9). We can then identify this single spiral with $Z(I, \dot{I})$. Taking the product with $1_{C}$ we obtain an action map from $\left(X\left(A, A_{0}\right), T\left(A, A_{0}\right)\right)$ to $(X(I, \dot{I}), T(I, \dot{I}))$. Thus, part (c) of Theorem 3.1 shows that we need only prove the result for the case $\left(A, A_{0}\right)=(I, \dot{I})$.

Now let $R$ be a surjective relation on a finite set $W$. We must construct a $\phi: X(I, \dot{I}) \rightarrow W$ which maps $T(I, \dot{I})$ to $R$.

Think of the set $W$ as the vertices of a finite directed graph with an edge from $a$ to $b$ iff $(a, b) \in R$. A bi-infinite $R$ chain is a sequence $\left\{a_{i}: i \in \mathbb{Z}\right\}$ with $\left(a_{i}, a_{i+1}\right) \in R$ for all $i$. We can extend any pair $\left(a_{0}, a_{1}\right) \in R$ to a bi-infinite $R$ chain which is eventually periodic as $i \rightarrow+\infty$ and as $i \rightarrow-\infty$. For the positive side just move forward along the graph and when some vertex is hit a second time just repeat the loop ad infinitum. For the negative side move backwards along the graph. Notice that since $R$ is a surjective relation we can arrive at and leave from any vertex. Because the map from $\mathbb{Z}$ to the chain is eventually periodic at each end, it extends (by the universality of $\Theta$ ) to a continuous map from the spiral $\Sigma$ into $W$ which maps $\tau$ onto a subset of $R$.

With finitely many chains we can cover $R$ and so we can map onto $R$ with a finite number, $N$, of disjoint spirals. Choose a decomposition of $C$ with $N$ pieces labeled by the chains. On each piece $Z(I, \dot{I}) \times V$, first project to the spiral $Z(I, \dot{I})$ and then map to the associated chain. The concatenation of these mappings is the required map $\phi$.

If $T \in \mathcal{H}(X)$ has dense type, then it has periodic orbits of all periods as factors and so the restriction of $T$ to any closed, invariant subset has the universal adding machine as a factor; see Corollary 8.12 of $[\mathrm{AHK}$. Since it has any finite union of finite spirals as a factor as well one might suppose that any $(X, T)$ of dense type (on a Cantor space $X)$ has $(X(I, \dot{I}), T(I, \dot{I}))$ as a factor. However, there exist homeomorphisms of dense type with only countably many adding machines. To see this, first identify all of the left end adding machines in $X(I, \dot{I})$ together via $\zeta$ and then similarly identify all the right ends together. We obtain a Cantor space homeomorphism $\left(Y_{0}, S_{0}\right)$ with two adding machines: the common alpha limit set and the common omega limit set of each of the uncountable sets of spirals. Taking the product with the identity map on the one-point compactification of $\mathbb{Z}$ we obtain a system $(Y, S)$ to which the argument in Proposition 3.2 applies. Hence, it is of dense type.

On the other hand, we can extend the construction of a spiral by allowing the limit set to be any topologically transitive (or even chain transitive) homeomorphism of the Cantor set and we can put these together to build the analogues of $\left(X\left(A, A_{0}\right), T\left(A, A_{0}\right)\right)$. If each of the limit sets has the universal adding machine as a factor, then these new homeomorphisms will all have the original $(X(I, \dot{I}), T(I, \dot{I}))$ as a factor and so they will all be of dense type.

Thus, the set of homeomorphisms of dense type contains a rich variety of distinct examples. Among these, the Special Homeomorphisms are indeed special. 
Theorem 3.3. For a Cantor space $X$ the set $\mathcal{S}_{X}$ of Special Homeomorphisms on $X$ is a single orbit which is a dense $G_{\delta}$ subset of $\mathcal{H}(X)$. In particular, the Special Homeomorphisms are of dense type, i.e. $\mathcal{S}_{X} \subset \mathcal{T}_{X}$. The union of the remaining orbits is the complement $\mathcal{H}(X) \backslash \mathcal{S}_{X}$ and so is of first category in $\mathcal{H}(X)$.

Proof. A Special Homeomorphism $T \in \mathcal{S}_{X}$ is isomorphic to the canonical Special Homeomorphism $T(D, C)$ on $X(D, C)$. So from Proposition 3.2 it follows that $T$ is of dense type on $X$, i.e. $T \in \mathcal{T}_{X}$. As noted above $\mathcal{S}_{X}$ is a single orbit because the Special Homeomorphisms on $X$ are isomorphic to one another. Since the Special Homeomorphisms are of dense type, $\delta_{X}$ is dense in $\mathcal{H}(X)$.

By Corollary 2.3 the Special Homeomorphisms are exactly those elements of $\mathcal{H}(X)$ which satisfy the Lifting Property. We complete the proof by showing that the Lifting Property defines a $G_{\delta}$ subset of $\mathcal{H}(X)$. For any continuous surjection $\phi: X \rightarrow \mathcal{W}_{n}$ and any positive rational $\epsilon$ let

$$
\mathcal{G}[\phi, \epsilon] \quad=_{\text {def }} \quad\left(\mathcal{H}(X) \backslash \mathcal{H}\left[\phi, R_{n}\right]\right) \cup\left(\bigcup_{\rho, k} \mathcal{H}\left[\rho, R_{n+k}\right]\right)
$$

where the union is taken over all positive integers $k$ and continuous surjections $\rho$ : $X \rightarrow \mathcal{W}_{n+k}$ with mesh less than $\epsilon$ and which satisfy $\xi \circ \rho=\phi$ where $\xi: \mathcal{W}_{n+k} \rightarrow \mathcal{W}_{n}$ is the projection in the inverse system $\left\{\mathcal{W}_{i}, \xi\right\}$. Recall that $\xi$ maps $R_{n+k}$ to $R_{n}$ and so $\mathcal{H}\left[\rho, R_{n+k}\right] \subset \mathcal{H}\left[\phi, R_{n}\right]$ when $\xi \circ \rho=\phi$.

As a union of clopen sets each $\mathcal{G}[\phi, \epsilon]$ is open. As a compact metric space has only countably many clopen decompositions, there are only countably many continuous maps $\phi: X \rightarrow \mathcal{W}_{n}$. Of course, there are only countably many positive rationals. Hence, $L=\bigcap_{\phi, \epsilon} \mathcal{G}[\phi, \epsilon]$ is a $G_{\delta}$ set. If $T \in L$, then for any $\phi$ either $\phi$ does not map $T$ to $R_{n}$, or if it does, then for any positive $\epsilon$ it admits a lifting, $\rho$, with mesh less than $\epsilon$. That is, $L$ is exactly the set of homeomorphisms on $X$ which satisfy the Lifting Property.

Distinct orbits are disjoint and so if $T$ is not in $\mathcal{S}_{X}$, then its entire orbit lies in the complement, which is of first category.

\section{A locally compact Rohlin group}

In this section we construct a locally compact group with the Rohlin Property. That is, the adjoint action of the group on itself is topologically transitive.

Suppose $\left\{K_{n}\right\}$ is an increasing sequence of topological spaces. That is, the topology on $K_{n}$ agrees with the subspace topology induced from the spaces later in the sequence. The inductive topology on $K={ }_{\text {def }} \bigcup_{n} K_{n}$ is the largest topology such that each inclusion is continuous. That is, $A \subset K$ is open (or closed) iff the intersection $A \cap K_{n}$ is open (resp. closed) in $K_{n}$ for every $n$.

Proposition 4.1. Let $\left\{K_{n}\right\}$ be an increasing sequence of compact, metrizable topological groups such that $K_{n}$ is a clopen subgroup of $K_{n+1}$ for all $n$. Give the union $K$ the inductive topology and introduce the unique multiplication operation which extends the group multiplication on the $K_{n}$ 's.

(a) $K$ is a locally compact, metrizable topological group and each $K_{n}$ is a clopen subgroup of $K$.

(b) The open subsets of the $K_{n}$ 's together form a basis for the topology on $K$.

Proof. If $A$ is an open subset of $K_{i}$ for some $n$, then since each $K_{n}$ is a clopen subset of its successors, it follows that $A$ is an open subset of $K_{i+k}$ for $k=0,1, \ldots$. 
Furthermore, $A \cap K_{j}$ is open in $K_{j}$ for all $j<i$. Hence, $A$ is an open subset of $K$. On the other hand, if $A \subset K_{i}$ is open in $K$, then $A=A \cap K_{i}$ is open in $K_{i}$. Furthermore, the same results hold when we replace open by closed throughout.

In particular, each $K_{n}$ is a clopen subset of $K$. Since each $K_{n}$ is compact, $K$ is locally compact, and condition (b) is clear as well. As it is the countable union of open sets each of which is second countable, $K$ is second countable and hence metrizable.

Since the multiplication restricts to a continuous function on the open sets $K_{n} \times$ $K_{n} \subset K \times K$, it follows that multiplication is continuous on $K$. Similarly, this holds for the inversion function and so $K$ is a topological group.

Let $\mathcal{J}=\left\{J_{i}: i=1,2, \ldots\right\}$ be a sequence of nonempty, finite subsets of $\mathbb{N}$ with strictly increasing cardinality which together partition $\mathbb{N}$. Let

$$
J^{j} \quad=\operatorname{def} \bigcup_{i=1}^{j} J_{i} .
$$

Let $S_{i}$ be the symmetric group on $J_{i}$, i.e. the finite group of permutations of the finite set $J_{i}$, and let $S^{i}$ be the symmetric group on $J^{i}$. For $k=0,1, \ldots$ we can concatenate permutations on $J^{i}, J_{i+1}, \ldots$, and $J_{i+k}$ to obtain a permutation on $J^{i+k}$. Thus, we will regard the product $S^{i} \times S_{i+1} \times \ldots \times S_{i+k}$ as a subgroup of $S^{i+k}$.

Now for $n=1,2, \ldots$ define the infinite product

$$
K_{n} \quad=_{\text {def }} \quad S^{n} \times S_{n+1} \times S_{n+2} \times \ldots .
$$

As the product of finite groups this is a topological group with underlying space a Cantor space. Using the identification via concatenation as above we can regard $K_{n}$ as a subgroup of the group of all permutations of the countable set $\mathbb{N}$. That is, $K_{n}$ consists of those permutations of $\mathbb{N}$ which preserve beyond $n$ the interval structure of $\mathcal{J}$. With this identification, each $K_{n}$ is a clopen subgroup of $K_{n+1}$ and so we can apply the proposition above to define the locally compact, metrizable group $K=\bigcup_{n} K_{n}$.

If $a \in K_{n}$, then we let $a^{(n)} \in S^{n}$ be the permutation of $J^{n}$ which is the restriction of $a$. It is the first coordinate of $a$ in the product given by (4.2). Since $K_{n} \subset K_{n+k}$ for $k=0,1, \ldots$ we obtain a definition for $a^{(n+k)} \in S^{n+k}$ as well.

The following implication is obvious, but important.

For $n=1,2, \ldots$ and $k=0,1, \ldots$,

$$
a \in K_{n}, b \in K_{n+k} \text { and } a^{(n+k)}=b^{(n+k)} \quad \Longrightarrow \quad b \in K_{n} .
$$

That is, if $b$ preserves $\mathcal{J}$ beyond $n+k$ and agrees with $a$ up to $n+k$, then it preserves $\mathcal{J}$ beyond $n$ because $a$ does.

For $n=1,2, \ldots$ and $\pi \in S^{n}$ define

$$
K(\pi) \quad{ }_{\text {def }} \quad\left\{a \in K_{n}: a^{(n)}=\pi\right\} .
$$

Proposition 4.2. $\quad$ (a) The $K(\pi)$ 's form a countable basis of clopen sets for the topology of $K$.

(b) If $a \in K_{n}$, then for $k=0,1, \ldots$ each $K\left(a^{(n+k)}\right)$ is contained in $K_{n}$. The sequence of clopen sets $\left\{K\left(a^{(n+k)}\right)\right\}$ is decreasing and forms a basis for the neighborhoods of a in $K$. 
Proof. Clearly $K(\pi)$ is an open subset of $K_{n}$. Since $K_{n}$ is an open subset of $K$ it follows that $K(\pi)$ is an open subset of $K$. If $b \in K\left(a^{(n+k)}\right)$, then $b \in K_{n}$ by (4.3). As $k$ tends to infinity, the sequence of clopens $K\left(a^{(n+k)}\right)$ decreases because $b^{(n+k+1)}=a^{(n+k+1)}$ implies $b^{(n+k)}=a^{(n+k)}$. Since the intersection is just $a$ they form a basis for the neighborhood system by compactness. This proves (b) which in turn implies that the $K(\pi)$ 's form a basis. There are only countably many finite permutations $\pi$.

Now for the key idea.

Suppose that $\beta: J^{n} \rightarrow J_{n+k}$ is an injective mapping. This requires that $k$ be large enough so the cardinality of $J_{n+k}$ is as large as that of $J^{n}$. Extend $\beta$ to a permutation $b$ of $\mathbb{N}$ by using $\beta^{-1}$ on $\beta\left(J^{n}\right)$ and the identity on $\mathbb{N} \backslash\left(J^{n} \cup \beta\left(J^{n}\right)\right)$. Clearly, $b$ is an element of order 2 in $K_{n+k}$. We will call $b$ the exchanger associated with $\beta$.

For $\pi \in S^{n}$ and $a \in K$ we will say that a contains a copy of $\pi$ if there exists an injection $\beta: J^{n} \rightarrow J_{n+k}$ such that on $J^{n}$,

$$
\beta \circ \pi=a \circ \beta .
$$

Clearly such an injection $\beta$ exists when for all $i$ the permutation $a$ contains in $J_{n+k}$ at least as many cycles of length $i$ as occur in $\pi$.

Lemma 4.3. If $\pi_{1}, \pi_{2} \in S^{n}$, then there exists $b \in K$ such that

$$
b K\left(\pi_{1}\right) b^{-1} \cap K\left(\pi_{2}\right) \neq \emptyset .
$$

Explicitly, if $a \in K\left(\pi_{1}\right)$ and a contains a copy of $\pi_{2}$, then bab $b^{-1} \in K\left(\pi_{2}\right)$ when $b$ is the associated exchanger and, furthermore, such a's always exist.

Proof. From the construction of $b$ associated with an injection $\beta: J^{n} \rightarrow J_{n+k}$ it is clear that $a \in K_{n}$ implies $b a b^{-1} \in K_{n}$, i.e. the $\mathcal{J}$ structure is still preserved beyond $n$. If, in addition, $a=\beta \circ \pi_{2} \circ \beta^{-1}$ on $\beta\left(J^{n}\right)$, then $b a b^{-1} \in K\left(\pi_{2}\right)$.

To construct such an $a$ choose an injection $\beta: J^{n} \rightarrow J_{n+k}$. Define the permutation $a$ of $\mathbb{N}$ as follows: on $J^{n}, a=\pi_{1}$ and on $\beta\left(J^{n}\right), a=\beta \circ \pi_{2} \circ \beta^{-1}$. Finally, let $a$ be the identity on $\mathbb{N} \backslash\left(J^{n} \cup \beta\left(J^{n}\right)\right)$.

For $\pi \in S^{n}$ let $G(\pi)$ denote the union of the orbits which pass through $K(\pi)$. That is,

$$
G(\pi) \quad=\operatorname{def} \bigcup_{b \in K} b K(\pi) b^{-1} .
$$

Theorem 4.4. For each $\pi \in S^{n}, G(\pi)$ is an open, dense conjugation-invariant subset of $K$. The dense $G_{\delta}$ set

$$
\mathcal{T}=\operatorname{def} \bigcap_{\pi} G(\pi)
$$

is exactly the transitive points for the adjoint action of $K$ on itself. In addition, if $a \in K$ and for every $n$ and every $\pi \in S^{n}$ a contains a copy of $\pi$, then $a \in \mathcal{T}$.

In particular, $K$ satisfies the Rohlin Property.

Proof. Given $\pi \in S^{n}$ and $a \in K_{m}$ and an arbitrarily large positive integer $k$ we must show that the neighborhood $K\left(a^{(m+k)}\right)$ meets $G(\pi)$. We can assume that $k$ is large enough that $m+k>n$ and so there exists a positive integer $p$ such that $m+k=n+p$. Choose $c \in K(\pi)$ so that $c \in K_{n}$. 
Let $\pi_{1}=a^{(m+k)}$ and $\pi_{2}=c^{(n+p)}$. Since $c^{(n)}=\pi, K\left(\pi_{2}\right) \subset K(\pi)$ by Proposition $4.2(\mathrm{~b})$.

Choose $a_{1} \in K\left(\pi_{1}\right)$ so that $a_{1}$ contains a copy of $\pi_{2}$. If $a$ contains such a copy, then choose $a_{1}=a$. In any case, such an $a_{1}$ exists by Lemma 4.3 , which also says that $b a_{1} b^{-1} \in K\left(\pi_{2}\right)$ for some $b \in K$. Hence, $a_{1} \in G(\pi)$.

This shows that each $G(\pi)$ is dense and that $a$ is in the intersection $\mathcal{T}$ when $a$ contains a copy of every finite permutation.

By the Baire Category Theorem, the set $\mathcal{T}$ is dense and so is nonempty, which is the Oxtoby Philosophy again. Alternatively, it is easy to construct an element $a \in K$ which contains a copy of every finite permutation.

A point $b$ is in $\mathcal{T}$ iff it lies in every $G(\pi)$ and so iff its orbit meets every $K(\pi)$. By Proposition 4.2 (a) the $K(\pi)$ 's form a basis and so $\mathcal{T}$ is the set of transitive points for the adjoint action.

A permutation $a$ of $\mathbb{N}$ which lies in $K$ decomposes $\mathbb{N}$ into disjoint finite cycles. If $a$ contains only finitely many cycles of length $i$, then no conjugate of it lies in $K(\pi)$ when the finite permutation $\pi$ contains more than this many cycles of length $i$. Thus, in order that $a$ lie in $\mathcal{T}$ it is necessary that $a$ contain infinitely many cycles of each length. Thus, the set $\tilde{K}$, consisting of permutations $a$ such that $a(j)=j$ for all sufficiently large $j \in \mathbb{N}$, is disjoint from $\mathcal{T}$. $\tilde{K}$ is a normal subgroup of $K$. It meets every $K(\pi)$ for every finite permutation $\pi$ and so is dense. We will now show that if the complement of the set of transitive points is dense and the group is locally compact and $\sigma$-compact, then there is no single dense $G_{\delta}$ conjugacy class. Thus, the group $K$ does not have the strong Rohlin Property exhibited in the previous section by the Polish group $\mathcal{H}(X)$ with $X$ a Cantor space.

Recall that a topological space $X$ is Baire when every countable family of open subsets dense in $X$ has a dense intersection, or, equivalently, when every first category subset of $X$ has empty interior. A topological group is Baire when the underlying space is.

Proposition 4.5. Let $\Phi: G \times X \rightarrow X$ be a topological action of a $\sigma$-compact topological group $G$ on a Baire space $X$. For $x \in X$ let $\Phi_{x}: G \rightarrow X$ be the map given by $g \mapsto g x$. Assume that for some $x \in X$ the orbit $G x=\Phi_{x}(G)$ is not of first category. The map $\Phi_{x}$ is then an open map and $G x$ is an open subset of $X$ which is locally compact in the subspace topology. If, in addition, the action $\Phi$ is topologically transitive, then $G x$ is dense and this dense open orbit is exactly the set of transitive points for the action.

Proof. Let $C$ be a compact subset of $G$. If the compact set $C x \subset X$ has empty interior, then it is nowhere dense. If this is true for all such $C$, then $G x$ is of first category because $G$ is $\sigma$-compact. Hence, there exists a compact set $C \subset G$, an open set $U \subset X$ and an element $g \in C$ such that $g x \in U$ and $U \subset C x$. For any $h \in G, h x \in h g^{-1} U$ and $h g^{-1} U \subset h g^{-1} C x$. Thus, every point of $G x$ has a compact neighborhood in $X$ which is a subset of $G x$. Thus, $G x$ is open and locally compact.

To prove that the map $\Phi_{x}$ is open it suffices to show that if $V$ is a neighborhood of the identity $e$ in $G$, then $V x$ is a neighborhood of $x$ in $X$. Choose $V_{1}$ to be a closed neighborhood of $e$ such that $V_{1}=V_{1}^{-1}$ and $V_{1}^{2} \subset V$. By compactness we can choose a finite subset $F$ of $G$ so that $\left\{f V_{1} \cap C: f \in F\right\}$ is a cover of $C$ by compact sets. As above, since $C x$ is not of first category, some $f V_{1} x$ has nonempty interior 
and so they all do by translation. In particular, $V_{1} x$ is a neighborhood of $f x$ for some $f \in V_{1}$. Thus, $V x \supset f^{-1} V_{1} x$ is a neighborhood of $x$.

If, in addition, the action is topologically transitive, then every nonempty invariant open subset of $X$ is dense and contains every transitive point. In particular, $G x$ is dense and contains all the transitive points. Since it is the (dense) orbit of each of its points, the points of $G x$ are all transitive points.

Corollary 4.6. Assume that $G$ is a $\sigma$-compact, Baire group. Then $G$ is locally compact and every conjugacy class of $G$ is either of first category or is open. If, in addition, $G$ satisfies the Rohlin Property, then either every conjugacy class is of first category or there is a unique open conjugacy class which is dense and which consists of all of the conjugacy transitive points of $G$. In particular, if there is a dense $G_{\delta}$ conjugacy class, then this conjugacy class is open.

Proof. For the first result apply Proposition 4.5 to the action of $G$ on itself by left translation, i.e. $\Phi$ is the multiplication map. Every orbit is the entire space and so is not of first category. Hence, the space is locally compact.

The remaining results follow immediately from Proposition 4.5 applied to the adjoint action of $G$ on itself, together with the observation that a dense $G_{\delta}$ subset of a Baire space is not of first category.

Problem 4.7. Is there a nontrivial Polish topological group with a dense, open conjugacy class? If so, can the group be chosen to be locally compact?

In considering this problem it is suggestive to recall that in $\mathrm{Os}$, Osin constructed an example of a finitely generated (discrete) group such that the complement of the identity is a single conjugacy class.

\section{REFERENCES}

[A] E. Akin, Good measures on Cantor space, Trans. Amer. Math. Soc. 357, (2005), 26812722. MR2139523 (2006e:37003)

[AHK] E. Akin, M. Hurley and J. Kennedy, Dynamics of topologically generic homeomorphisms, Mem. Amer. Math. Soc. 164, (2003), no. 783. MR1980335 (2004j:37024)

[GW] E. Glasner and B. Weiss, The topological Rohlin property and topological entropy, Amer. J. Math. 123, (2001), 1055-1070. MR1867311 (2002h:37025)

[KR] A. S. Kechris and C. Rosendal, Turbulence, amalgamation and generic automorphisms of homogeneous structures, Proc. London Math. Soc. 94, (2007), 302-350. MR2308230

[Os] D.V. Osin, Small cancellations over hyperbolic groups and embedding theorems, arXiv:math.GR/0411039v1, 2 Nov 2004.

[O] J. Oxtoby, Measure and category ( $2^{\text {nd }}$ Ed.) Springer-Verlag, Berlin, 1980. MR584443 (81j:28003)

Mathematics Department, The City College, 137 Street and Convent Avenue, New York, New York 10031

E-mail address: ethanakin@earthlink.net

Department of Mathematics, Tel Aviv University, Tel Aviv, Israel

E-mail address: glasner@math.tau.ac.il

Institute of Mathematics, Hebrew University of Jerusalem, Jerusalem, Israel

E-mail address: weiss@math.huji.ac.il 